\title{
The WHO-HPH recognition project: fast- track implementation of clinical health promotion - a protocol for multi-center RCT
}

\author{
Hanne Tønnesen ${ }^{1,2,3}$, Jeff Kirk Svane ${ }^{2}$, Oliver Groene ${ }^{4}$, Shu-Ti Chiou ${ }^{5}$
}

\begin{abstract}
Background Clinical health promotion comprises services delivered in health care to address daily smoking, risky alcohol use, overweight/obesity, malnutrition and physical inactivity. Clinical health promotion significantly improves treatment results and patient safety. Accordingly, it is a core component of overall quality in hospitals. To further implementation, the World health Organization and the International Network of Health Promoting Hospitals and Health Services have developed standards and models based on quality management and accreditation/recognition strategies. However, these implementation strategies have only been sparsely investigated in randomised trials. The aim of the present study described in this protocol article is to evaluate the effect of a fast-track program for implementation on delivery of clinical health promotion services and the associated health gain of patients and staff.

Methods Clinical hospital departments are recruited through an open call. The departments are randomized to either fasttrack implementation or to continue their usual implementation routines. The intervention group departments measures baseline, produce a quality plan on own results, implement for 1 year and then re-measure. The control group departments wait 1 year following allocation to perform just the baseline measurement.

The primary outcome is physical, mental and social health status of patients and staff. The secondary outcome is clinical health promotion service delivery to address patients' needs thereof. The data will be analyzed as intention-to-treat.

Discussion Today, a total of 48 clinical departments from 11 countries/regions are included. This is the first study ever to evaluate the health effect of a fast-track implementation program for clinical health promotion.
\end{abstract}

doi.org/10.29102/clinhp.16003

\section{About the AUTHORS}

${ }^{1}$ Clinical Health Promotion Centre, Health Sciences, Lund University, Sweden. ${ }^{2}$ Clinical Health Promotion Centre, Bispebjerg \& Frederiksberg Hospital, Copenhagen University Hospitals, Denmark.

${ }^{3}$ Health Science, University of Southern Denmark. ${ }^{4}$ OptiMedis AG, Germany. ${ }^{5}$ School of Medicine, National Yang-Ming University, Taiwan.

Contact: Jeff Kirk Svane Jeff.Kirk.Svane@RegionH.dk

\section{Background}

It is well-known that the overall burden of disease is heavily influenced by smoking, alcohol, unhealthy nutrition and physical inactivity (1). As a result, there is widespread need for health promotion activities throughout society; in families and at work places, in schools and in institutions and other settings. The need also exists in health care settings, and here too, it is quite substantial in scale; $80-90 \%$ of patients have risky lifestyle factors that aggravate their patient pathways (2). Additionally, strong evidence highlights the significant benefits of health promotion intervention in patient pathways. Applying health promotion activities directly in health care settings means supporting patients to quit daily smoking, control alcohol-drinking and nutrition, and maintain a healthy level of physical activity. Interventions addressing this have been termed "clinical health promotion" (CHP) $(3 ; 4)$, and have been proven to increase patient safety and improve treatment results in the areas of surgery, internal medicine, obstetrics and psychiatry (5-9) and be cost-effective (10). Consequently, CHP is a core element in high quality health care (11-13) in line with other evidence-based clinical interventions.

To facilitate CHP implementation, the World Health Organization (WHO) and the International Network of Health Promoting Hospitals and Health Services (HPH) have developed and validated a set of standards and indicators $(14 ; 15)$ following the International Society for Quality in Health Care criteria (16). Two supplementary models used for practical clinical documentation of CHP needs and services delivered were also developed and validated internationally $(17 ; 18)$. These models help clinicians and managers with implementation of CHP: namely diagnosing the patients' needs for CHP (e.g daily smoking) and delivering the related interventions (e.g. smoking cessa- 


\section{Research and Best Practice}

tion counselling). These tools have been implemented completely or in part in national or regional health services in several countries including Sweden, Denmark and Ireland.

A general challenge in health care settings is real-life implementation of new evidence for the benefit of patients. This is also the case for CHP (19;20). Accordingly, it is necessary to develop, describe and evaluate a fast-track implementation program to increase integration speed. Such a fast-track program should build on the commonly used strategies for implementation; such as quality management, staff training and recognition/accreditation.

However, these commonly used strategies have only been sparsely investigated in high quality research designs like randomised trials. Until now, just one study has evaluated the effect of accreditation, but without including health measurements as an outcome (21).

\section{Methods/design}

The aim of the present study is to evaluate the effects of a fast-track program for implementation of CHP on frequency of delivery of CHP services and on health gains of patients and staff.

The main study hypothesis is that clinical hospital departments allocated to the fast-track program for CHP will display improved health gains for their patients and staff after one year, compared to the control group departments that continue usual implementation routines. The secondary hypothesis is that the intervention group departments will display more frequent delivery of CHP services.

The study is a multi-national, randomized trial with two arms. The clinical department is the unit of randomization and measurement - not individual patients. This approach is necessary to test the program's effect on overall organizational performance of the clinical department.

The study takes place in various types of surgical, medical and psychiatric hospital departments.

\section{Material}

There is a total of 48 clinical departments participating, 21 in Taiwan, 8 in the Czech Republic, 4 in Croatia, 4 in Thailand, 3 in Slovenia, 2 in Estonia, 2 in Japan, 1 in Denmark, 1 in Malaysia, 1 in Canada and 1 in Indonesia. Inclusion is conducted through an open call for participation to all 29 National/Regional HPH Net- work Coordinators, who are asked to present the call to their member hospitals. The call is repeated at the International HPH Conferences each year and in the related scientific journal of Clinical Health Promotion (www.clinhp.org). Participation is approved by each participating hospital's CEO, the head of the participating department and the National/Regional HPH Coordinator.

\section{Inclusion criteria}

All clinical hospital departments treating inpatients and/or outpatients are eligible for inclusion; university as well as non-university hospitals with rural, mixed or urban catchment areas, but each included hospital can have only one clinical department participate in the study.

\section{Exclusion criteria}

Palliative care departments, paediatric departments, nursing homes, non-hospital clinics, and primary care facilities are excluded, as the WHO standards and the other tools are not yet validated for these types of clinical settings.

\section{Processes, interventions and comparisons}

The randomization is computerised using blocks of unknown sizes between 3 to 8 . Stratification is done for each participating regional/national HPH Network. The randomisation is performed by an independent researcher, who is not otherwise involved with the study. Randomization envelopes are opaque and sealed by the independent researcher. The allocation was video recorded. The study is by nature not blinded, but all statistical analyses are undertaken by a blinded, independent researcher not otherwise involved in the project.

\section{Outcomes}

The outcomes were measured and analysed at department level. The health gain among patients and staff is calculated via health related quality of life using SF36 health questionnaires for self-reporting on eight dimensions of physical and mental health. This questionnaire has been translated and validated worldwide (22).

The health promotion deliveries are measured by:

- self-assessment tools: WHO-HPH standards for health promotion in hospitals with a total of 40 measurable elements and 18 indicators provided in a manual (11), the 9-question HPH DATA Model (17) and the 16-deliverable services HPH DOC-ACT Model (18).

- $\quad$ internal audit of 50 medical records, where the 


\section{Research and Best Practice}

HPH DATA Model is used for practical clinical assessment of patient needs for CHP services and the HPH DOC-ACT Model for assessment of CHP service delivery.

\section{Intervention}

After allocation, the intervention group departments (IGD) measure their baseline CHP performance and health status of patients and staff (see data collection, below). After measuring, IGD produce a quality plan for CHP according to the fast-track program and based on analysis of own baseline results. The quality plan is then implemented over 1 year, at which point CHP performance and health status are re-measured in the same way as for baseline - and followed by a site visit for validation (Figure 1).



\section{Comparison}

The control group departments (CGD) wait 1/2-1 year after allocation, and they then perform their measurement similar to baseline of IGD. The waiting period reduces possible contamination from results on usual implementation routines. All CGD are offered support to use the fast-track implementation program after the IGD have finalized their implementation period.

\section{Data collection}

All data collected are anonymized locally at the source. No type of person-identifiable information is transferred. Reported data from participating centres is in electronic or paper format. Upon receipt by research group and initial data validation, all data is entered into the electronic project databases using numeric codes. All data is stored on an internal hospital drive, secured by Capital Region Denmark CIMT to avoid risk of data loss. Only project staff and researchers have access to the anonymous database and archives.

\section{SF-36}

The health status at each department is based on data collected from up to 200 consecutive patients, who visit the department in the month immediately prior to inclusion in the study. If 200 patients are not seen in that particular month, the department only includes the patients of that month. Data from all staff employed in the department (at any point) during the same month are also collected.

\section{WHO HPH standards}

The self-assessment of CHP delivery is done with a total of 40 measurable elements and 18 indicators provided in the WHO HPH standards manual (11) (Table 1).

The WHO HPH standards cover 5 areas:

1. policy for health promotion

2. patient needs assessment

3. patient information and intervention

4. creation of a healthy workplace

5. continuity and collaboration with outside providers and other sectors

\section{HPH DATA Model}

The HPH Data Model is used for practical clinical assessment and documentation of patient needs for CHP services. It fits directly into WHO HPH standard 2 (above), and precedes the HPH DOC-ACT Model below. It is used for internal audit of 50 consecutive and anonymized medical records on patients in contact with IGD and CGD. The period assessed is one month before inclusion corresponding to the measurement of 


\section{Research and Best Practice}

Table 1 WHO-HPH standards and measurable elements

\section{Standard 1: Management Policy}

1.1.1. Aims and mission include HP

1.1.2. Minutes reaffirm agreement $w \mathrm{HPH}$

1.1.3. Quality/business plans include HP

1.1.4. Personnel and functions ID'ed for HP

1.2.1. There is a budget for $\mathrm{HP}$

1.2.2. HP procedures available

1.2.3. HP structures and facilities can be ID'ed

1.3.1. HP intervention data captured

1.3.2. Assessment of HP established

\section{Standard 2: Patient Assessment}

2.1.1. Guidelines to ID lifestyle risk exist

2.1.2. Guidelines have been revised

2.1.3. Guidelines to ID HP needs exist

2.2.1. Assessment is documented

2.2.2. Guidelines for reassessing $H P$ needs

2.3.1. Info from referring $D R$ available in $M R$

2.3.2. MR documents social/cultural background

Standard 3: Patient Information \& Intervention

3.1.1. Information given is recorded in MR

3.1.2. HP activities are documented in MR

3.1.3. PT satisfaction assessment integrated in QM

3.2.1. General health information is available

3.2.2. Info about highrisk diseases is available

3.2.3. Information on PT organizations available

\section{Standard 4: Healthy Workplace}

4.1.1. Working conditions comply w N/R directives

4.1.2. Staff comply w health and safety

4.2.1. Intro training on HP policy given to new staff

4.2.2. Staff aware of HP policy

4.2.3. HP performance appraisal system exists

4.2.4. Practices made by multidisciplinary teams

4.2.5. Staff involved in policy-making

4.3.1. Policies on health issues avaliable for staff

4.3.2. Smoking cessation programmes offered

4.3.3. Annual staff surveys are carried out

Standard 5: Continuity and Cooperation

5.1.1. Regional policy taken into account

5.1.2. List of partners avaliable

5.1.3. Collaboration based on regional health plan

5.1.4. Plan for collaboration w partners avaliable

5.2.1. Follow-up instructions given

5.2.2. Procedure for info exchange exists

5.2.3. Receiving organization gets info on $\mathrm{PT}$

5.2.4. Rehab plan documented in MR

World Health Organization (2010). Implementing health promotion in hospitals: manual and self-assessment forms. (11)
SF-36 above. It continues backwards until 50 medical records are obtained. The HPH DATA Model comprises 9 questions that cover the 5 main risk factors (smoking, risky drinking, overweight/obesity, malnutrition and physical inactivity) influencing the treatment results of the patients. The model has been shown to be easy to understand and use, with a low inter-variation in the clinical setting (17), (table 2).

Table 2 HPH Data Model: Document risk in medical records

$$
\text { Yes/No Risk? }
$$

\section{A. Risk of malnutrition}

A1. Does patient have a $\mathrm{BMI}<20.5$ ?

A2. Has patient suffered from weight-loss in the past month?

A3. Has patient suffered from decreased food intake in the last wk.?

A4. Is patient severely ill (sepsis, burns, etc.)?

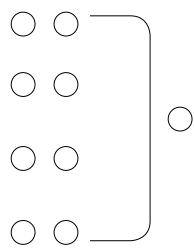

\section{B. Overweight}

B1. Does patient have a $\mathrm{BMI}>25$ ?

B2. Is patient's waist-measure $>80 \mathrm{~cm}(\mathrm{~W})$ or $94 \mathrm{~cm}(\mathrm{M})$ ?

\section{Physical inactivity}

C1. Is patient physically active $<1 / 2$ hour / day?

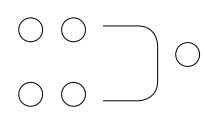

\section{Smoking}

D1. Does patient smoke daily?

\section{E. Drinking}

E1. Does patient drink > 14 drinks/wk (W) or $21(\mathrm{M})$ ?

Note: YES to ANY of the questions in group (A, B, C, D or E) equals RISK

\section{HPH DOC-ACT Model}

This model is used for assessment and documentation of patient-related CHP service deliveries, either as short face-to-face intervention or as the longer and more intensive interventions with repeated meetings. This model fits into standard 3 (above), and it follows the HPH DATA Model. The model includes 2x8 CHP activities, table 3. Clinical specialists responsible for coding treatment in the clinical setting have found the model understandable, applicable and adequate (18). The data collection is performed as described above for the HPH DATA Model. 


\section{Research and Best Practice}

Table 3 HPH Doc-Act Model: Documentation of Clinical Health Promotion activities in medical records

\begin{tabular}{|c|c|}
\hline $\begin{array}{l}\text { Counselling or motivational interviewing done } \\
\text { regarding: }\end{array}$ & DRG Code \\
\hline Smoking & BQFS01 \\
\hline Alcohol & BQFSO2 \\
\hline Nutrition & BQFSO3 \\
\hline Physical activity & BQFSO4 \\
\hline Psycho-social relation & BQFS05 \\
\hline Other risk factors & BQFSO6 \\
\hline Integrated counselling (consisting of several factors) & BQFS19 \\
\hline \multicolumn{2}{|l|}{$\begin{array}{l}\text { Intervention, rehabilitation or after-treatment } \\
\text { done regarding: }\end{array}$} \\
\hline Tobacco cessation & BQFT01 \\
\hline Alcohol intervention & BQFT02 \\
\hline Nutrition & BQFT03 \\
\hline Physical activity & BQFT04 \\
\hline Psycho-social support & BQFT05 \\
\hline Medicine after-treatment & BQFT06 \\
\hline Patient education & BVDY04 \\
\hline $\begin{array}{l}\text { Integrated rehabilitation (consisting of several } \\
\text { elements) }\end{array}$ & BQFT19 \\
\hline \multicolumn{2}{|c|}{$\begin{array}{l}\text { Note: DRG codes from Danish National Board of Health used here for } \\
\text { illustration purposes. } \\
\text { In Denmark, the reimbursement for each resembles ordinary visit to } \\
\text { primary care or out-patient clinic. } \\
\text { www.medinfo.dk }\end{array}$} \\
\hline
\end{tabular}

\section{Quality plan}

The template for making the required CHP quality plan for fast track implementation follows the traditional instructions for quality assurance and is shown in figure 2. The department's own data is the base for formulating the quality plan, milestones, actions and 1-year timeline. Minor adjustments to the plan are allowed and often necessary according to changes in hospital structure, patient group and staff in order to reach the milestones (Figure 2).

\section{Follow-up}

After the 1-year implementation, the baseline measurements are repeated for IGD, but this time covering the period of the month immediately following the 1-year implementation.

\section{Data validation through site visit}

The study participation for IGD concludes with a site visit that takes place after collection of follow-up data. The site visit focuses on validation of results on the WHO-HPH standards. The visit entails interviews with the staff and patients as well as an external audit of about 5 random medical records together with the local medical staff (and a trained interpreter in case of non-English language) to validate the internal data collection. At the visit, a certificate is issued outlining fulfilment of the WHO HPH standards, exclusively.

Figure 2 Template for Quality Plan
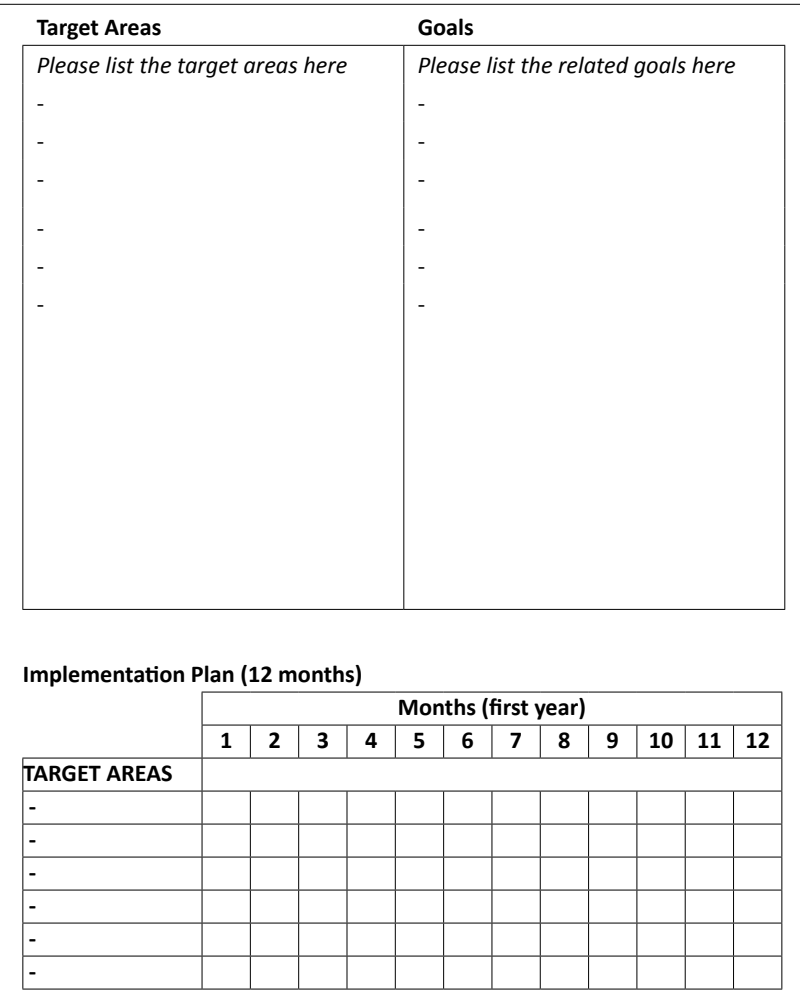


\section{Research and Best Practice}

\section{Statistical analyses}

The analyses will be conducted at department level and comparisons are made between IGD and CGD using intention-to-treat. The data is presented as median and range (but also as mean and SD for easy use in case of future meta-analysis). The health status from SF-36 will be analyzed with Mann-Whitney test, while frequencies will be compared by Fisher's exact test. An external researcher blinded for group allocation will conduct all analyses on the anonymized data using StataCorp LP's STATA 14 statistics software. P-value below 0.05 is considered significant.

Since no studies have been performed hitherto on health gain in this context, it would be difficult to make a meaningful power calculation for this primary outcome. However, looking for a minimum relevant difference of about $1 / 3$, the calculation of the secondary outcome below should be sufficient - also for the health gain analyses. The power calculation of the secondary outcome is based on the literature, assuming that frequency of CHP service deliveries is no higher than $40 \%$ of the needed (control departments $\pi 2=0.4$ ) (18). With a minimum relevant difference in deliveries of CHP services of $30 \%(\delta=0.3)$ between the intervention and control groups, the expected outcome is $70 \%$ (intervention departments $\pi 1=0.7$ ). Accordingly, 40 departments would be needed in each arm, considering $80 \%$ power and $5 \%$ two-sided significance.

Nevertheless, full inclusion for this study has not been reached before a scheduled update and revision of the WHO standards in 2016.

\section{Organization}

Clinical Health Promotion Centre, Bispebjerg-Frederiksberg Hospital, Copenhagen University, is responsible for project organization, data collection and administration and is in close collaboration with the participating Coordinators from the National/Regional HPH Network, the hospital managements, the heads of departments and the local HPH Hospital Coordinators. The National/Regional HPH Coordinators and the hospital HPH Coordinators are responsible for project support, while the hospitals' managements and heads of department are responsible for local project progress, collection of data, quality plan and implementation.

The project is approved by Copenhagen University as part of the PHD study for Jeff Kirk Svane, who is supervised by Professor Hanne Tønnesen (Main Supervisor; Copenhagen) and Professor Shu-Ti Chiou (Co-Supervisor; Taipei). Professor Oliver Groene (London) is International Project Advisor.

\section{Financial and budgetary administration}

The IGD and CGD fund their own study participation, primarily the individual hospitals and/or the ministry of health (Taiwan and Czech Republic). Bispebjerg-Frederiksberg Hospital in Denmark and Region Skåne in Sweden fund the project administration and local research staff.

\section{Discussion}

This is going to be the first randomized study on a fasttrack implementation strategy to include health gain as an outcome in addition to service deliveries. The focus is on integrating health promotion into the clinical treatment in order to obtain better treatment results and prognoses on short as well as longer term. The improved outcomes of individual programs at patient-level are already shown for patients in e.g. internal medicine, surgery, psychiatry and obstetrics $(23 ; 24)$. The education of staff members to improve CHP service delivery, and regarding their own health as such, are also important in this work, along with collaboration across departments and sectors.

\section{Status}

Until now, the first baseline data on the WHO HPH standards have shown variation in fulfilment of the 40 elements between hospital departments (Taiwan and the Czech Republic), leaving room for improvement. Our main outcomes of health gain and CHP deliveries has not yet been published as the study has not finalised yet.

The first departments were recruited in 2012, and by October 2015, 48 departments had been included. At that time, it was made official that WHO Europe would conduct a 2016 update and revision of the WHO standards for health promotion in hospitals (11). Therefore, the inclusion for this present study was halted prematurely at the 48 already included departments (representing $60 \%$ of the intended 80 departments), since the WHO standards are a main tool used in the study's data collection.

A main issue has been logistics and time consumption around project agreement by departments, which had to be signed by the department head and hospital director before inclusion. Also, several delays with data submissions have been experienced.

\section{Bias and limitations}

While the real-life conditions set-up is a strength, it is a limitation that all clinical departments included in the study are from HPH Network member hospitals, which 
might reduce the generalization to non-HPH hospital settings. Furthermore, it should be noted that all data are collected by self-assessment and self-reporting, which may introduce selective reporting biases with overestimation of performance.

The reduced number of participants will introduce a high risk of a type-2 error, and thereby risk of overlooking results that could have been of significance if the planned sizeable study was completed. However, even a sizeable study could not reduce a risk of type-1 error, which would require repetition of the study. It is a strength to report the results in accordance with the level of randomization (i.e. at department level), thus avoiding the further bias introduced by potential cluster randomization.

\section{Plan}

During 2016, all remaining in-coming data from the hospital departments will be collected, analyses conducted and results made public.

\section{Abbrevations}

- CHP: Clinical health promotion (incl. smoking cessation support for daily smokers, alcohol cessation support for excessive drinkers, nutritional support for obese or malnourished patients and physical activity support for the physically inactive).

- CGD: Control group department

- HPH: The International Network of Health Promoting Hospitals and Health Services

- IGD: Intervention group department

- SF-36: The Short Form Health Survey with 36-items for patient-reported of patient health

- WHO: World Health Organization

\section{Ethical considerations}

Participation will not cause departments, their patients or their staff any risk. However, it will entail approximately 100 hours of staff time per participating IGD and 50 hours per CGD. This resource allocation seems ethically balanced by the potentially possible improvement in health gain and CHP deliveries for the benefit of the patients and staff. Participation in the study only takes place after informed consent before inclusion by the involved hospital management, the head of the participating department, the National/Regional HPH Coordinator, and the local hospital HPH Coordinator. Also, the utilized components are already validated and implemented or being implemented, in part of in full, in several hospitals all over the world (see www.hphnet.org).
Ethical approval for this study was obtained from the Scientific Ethical Committee in the Danish Capital Region (International Studies) and Danish Data protection Agency (J.nr.2012-41-0152). Approval is also obtained by all relevant national/regional/local ethical and research authorities (such as internal review boards) as per the regulations and requirements in each hospital/ region/nation.

The intervention is conducted without following any individual patient or staff member, with all data anonymized at the source (at data collection) before transferal and storage at WHO-CC in secured files with access for the research team only - in order to fully guarantee confidentiality and security.

\section{Competing interests}

The authors declare that they have no competing interests.

\section{Authors' contributions}

- HT: Initial idea, drafted protocol, revised and approved manuscript. International Principal Investigator.

- JKS: Drafted manuscript, approved manuscript and revised protocol. International Project Leader.

- OG: Revised and approved the protocol and manuscript. International Project Advisor.

- STC: Revised and approved the protocol and manuscript, Responsible for included departments and data collection in Taiwan. International co-supervisor for PHD Study.

All authors have made substantial contributions to conception, design and acquisition of data. All authors read and approved the final manuscript.

\section{Acknowledgements}

World Health Organization, Regional Office for Europe, International Network of Health Promoting Hospitals \& Health Services (HPH), Regional HPH Network of Taiwan, National HPH Network of the Czech Republic, National HPH Network of Slovenia, National HPH Network of Estonia, National HPH Network of Japan, HPH member hospitals in Croatia, HPH member hospitals in Denmark, National HPH Network of Montreal Canada, National HPH Network of Indonesia, HPH member hospitals in Malaysia, HPH member hospitals in Thailand. 


\section{Research and Best Practice}

\section{References}

(1) Vos T, Flaxman AD, Naghavi M, et al. Years lived with disability (YLDs) for 1160 sequelae of 289 diseases and injuries 1990-2010: a systematic analysis for the Global Burden of Disease Study 2010. Lancet 2012; 380:21632196.

(2) Oppedal K, Nesvåg S, Pedersen B, Skjøtskift S, Aarstad AK, Ullaland S, Pedersen KL, Vevatne K, Tønnesen H. Health and the need for health promotion in hospital patients. Eur J Public Health. 2011; 21:744-9

(3) Tønnesen H, ed. Good Clinical Health Promotion Practice. Clin Health Promot 2014; 39-40. http://www.clinhp.org/ifile/Vol4_Issue2_p39_40.pdf (4) Danish National Board of Health (2005). Terminologi: Forebyggelse, sundhedsfremme og folkesundhed. http://sundhedsstyrelsen.dk/ /media/ CA0B2ED9165F4C908DB3117BA4764058.ashx

(5) Anderson L, Taylor RS. Cardiac rehabilitation for people with heart disease: an overview of Cochrane systematic reviews. Cochrane Database Syst Rev 2014 Dec 12; 12:CD011273.

(6) Thomsen T, Villebro N, Møller AM. Interventions for preoperative smoking cessation. Cochrane Database Syst Rev. 2014 Mar 27; 3:CD002294

(7) Oppedal K, Møller AM, Pedersen B, Tønnesen H. Preoperative alcohol cessation prior to elective surgery.Cochrane Database Syst Rev. $2012 \mathrm{Jul}$ 11; 7:CD008343.

(8) Taylor G, McNeill A, Girling A, Farley A, Lindson-Hawley N, Aveyard P. Change in mental health after smoking cessation: systematic review and meta-analysis. BMJ 2014; 348:g1151; 1-22.

(9) Gade P, Lund-Andersen H, Parving HH, Pedersen O. Effect of a multifactorial intervention on mortality in type 2 diabetes. N Engl J Med 2008; 358:580-591.

(10) Pedersen B, Hansen PE, Tønnesen H. Scand-Ankle: Cost-effectiveness of Alcohol Cessation Intervention in Acute Fracture Surgery. Clin Health Promot 2014; 2 suppl:1-68.

(11) World Health Organization (2010). Implementing health promotion in hospitals: manual and self-assessment forms. WHO Regional Office for Europe, Copenhagen. http://www.euro.who.int/_data/assets/pdf_ file/0009/99819/E88584.pdf

(12) World Health Organization (2013). Health 2020: A European policy framework supporting action across government and society for health and well-being. World Health Organization Regional Office for Europe, Copenhagen. www.euro.who.int/_data/assets/pdf_file/0006/199536/ Health2020-Short.pdf

(13) World Health Organization (2013). Global action plan for prevention and control of non-communicable diesases 2013-2020. World Health Organization, Geneva. http://www.who.int/nmh/events/2013/revised_draft_ ncd_action_plan.pdf
(14) Groene O, Jorgensen SJ, Fugleholm AM, Garcia Barbero M. Standards for health promotion in hospitals: development and pilot test in nine European countries. Int J Health Care Qual Assur Inc Leadersh Health Serv 2005; 18:300-7.

(15) Groene O, Alonso J and Klazinga N. Development and validation of the WHO self-assessment tool for health promotion in hospitals: results of a study in 38 hospitals in eight countries. Health Promot Int. 2010; 25:221-9. (16) www.isqua.org

(17) Tønnesen H, Svane JK, Lenzi L, Kopecky J, Suurorg L, Bukholm IRK, Hsu ST, Hübner M, Krogerus S, Kellner-Rechberger S, Masiello MG and the HPH Networks in Tuscany, Italy; Spain; Ontario, Canada and Germany. Handling Clinical Health Promotion in the HPH DATA Model: Basic Documentation of Health Determinants in Medical Records of tobacco, malnutrition, overweight, physical inactivity \& alcohol. Clin Health Promot 2012; 2:5-11.

(18) Tønnesen H, Christensen ME, Groene O, O’Riordan A, Simonelli F, Suurorg L, Morris D, Vibe P, Himel S and Hansen PE. An evaluation of a model for the systematic documentation of hospital based health promotion activities: results from a multicentre study. BMC Health Services Research 2007; 7:145.

(19) Svane JK, Chiou ST et al. Integration of health promotion in clinical hospital departments: standards fulfilment, documentation of needs and service delivery. Clin Health Promot 2015; 1:11-17.

(20) Svane JK, Raisova B et al. Clinical Health Promotion in the Czech Republic: Standards Compliance and Service Provision. Clin Health Promot 2014; 4:15-21.

(21) Salmon JW, Heavens J, Lombard C, Tawrow P. The Impact of Accreditation on the Quality of Hospital Care: KwaZulu-Natal Province, Republic of South Africa. 2003. Quality Assurance Project, University Research Co., LLC. USA.

(22) McHorney, Colleen A.; Ware, John E.; Raczek, Anastasia E. The MOS 36Item Short-Form Health Survey (SF-36): II. Psychometric and clinical tests of validity in measuring physical and mental health constructs. Med Care 1993; 31:247-263.

(23) Tønnesen $\mathrm{H}$, ed. Clinical treatment + clinical health promotion = Better treatment results immediately. Clin health promot 2013; 3-4. http://clinhp. org/ifile/Vol4_Issue1_p3_4.pdf

(24) Tønnesen H, ed. Pregnant smokers: Room for improvement. Clin Health Promot 2015; 3-4. http://www.clinhp.org/ifile/Vol5_Issue1_p3.pdf 